\title{
The N-terminal Leucine-Rich Regions in Slit Are Sufficient To Repel Olfactory Bulb Axons and Subventricular Zone Neurons
}

\author{
Jin-hui Chen, ${ }^{1}$ Leng Wen, ${ }^{1}$ Sophie Dupuis, ${ }^{2}$ Jane Y. Wu, ${ }^{2}$ and Yi Rao ${ }^{1}$ \\ Departments of ${ }^{1}$ Anatomy and Neurobiology and ${ }^{2} P e d i a t r i c s$ and Molecular Biology and Pharmacology, Washington \\ University School of Medicine, St. Louis, Missouri 63110
}

The Slit proteins are a new family of secreted guidance cues involved in axon guidance and neuronal migration. Each mammalian Slit protein contains $>1400$ amino acid residues, with four leucine-rich regions (LRRs), nine epidermal growth factor repeats, a laminin G domain, and a C-terminal cysteine-rich domain. A receptor for Slit is the transmembrane protein Roundabout (Robo), whose extracellular part contains five Ig domains and three fibronectin type III repeats. We report here that the LRRs in Slit are sufficient for binding to the Ig domains of Robo. Mutant forms of Slit containing only the LRRs function as chemorepellents for axons projecting from the olfactory bulb both in vitro and in the telencephalon. The LRRs can repel neurons migrating from the anterior subventricular zone (SVZa) to the olfactory bulb in brain slices isolated from neonatal rodents. However, the LRRs do not show repulsive effects on the SVZa neurons migrating in collagen gels. Our results indicate that the same LRRs are sufficient for guiding both axon projection and neuronal migration and suggest that the other regions in the Slit proteins may be involved in regulating the diffusion and distribution of the Slit proteins. The fact that the same domains are involved in guiding axon projection and neuronal migration further strengthens the idea of a conserved guidance mechanism for these important processes.

Key words: neuronal migration; axon guidance; Slit; Robo; molecular cues; cell migration
The precise projection of axons and deposition of neurons are essential for the development of the nervous system. Several families of proteins function as guidance cues for a variety of axons and neurons (Tessier-Lavigne and Goodman, 1996; Raper and Tessier-Lavigne, 1999). The Netrins are chemoattractants for some axons and repellents for other axons (Kennedy et al., 1994; Serafini et al., 1994; for review, see Colamarino and TessierLavigne, 1995). Semaphorins are chemorepellents for most axons, although they are attractants for some axons (for review, see Culotti and Kolodkin, 1996; Van Vactor and Lorenz, 1999; Raper, 2000). Ephrins are cell surface molecules that function as contact-dependent repellents (for review, see Flanagan and Vanderhaeghen, 1998; Frisen et al., 1999; Holder and Klein, 1999; Mellitzer et al., 2000).

The Slit proteins are the newest family of neuronal guidance molecules. Mutations in the slit gene were first described in Drosophila (Nusslein-Volhard et al., 1984). Drosophila slit cDNA was cloned when Rothberg et al. (1988) screened for genes encoding epidermal growth factor (EGF) repeats. Although Drosophila slit was thought to play a role in cell differentiation in the nervous system (Rothberg et al., 1988, 1990), recent work in Drosophila has shown that it is not involved in cell differentiation but rather in axon guidance (Battye et al., 1999; Kidd et al., 1999). Drosophila slit plays an important role in preventing commissural axons that have crossed the midline from recrossing the midline (Kidd et al., 1999). In the vertebrate spinal cord, Slit can repel motor axons (Brose et al., 1999) and can also promote branching

Received Aug. 22, 2000; revised Nov. 6, 2000; accepted Nov. 14, 2000.

We are grateful to the National Institutes of Health, the John Merck Fund, the Klingenstein Foundation, and the Leukemia Foundation of America for support.

Correspondence should be addressed to Yi Rao, Department of Anatomy and Neurobiology, Washington University School of Medicine, Box 8108, 660 South Euclid Avenue, St. Louis, MO 63110. E-mail: raoyi@thalamus.wustl.edu.

Copyright (C) 2001 Society for Neuroscience $0270-6474 / 01 / 211548-09 \$ 15.00 / 0$ of axons from the dorsal root ganglia (Wang et al., 1999). In the mammalian olfactory system, Slit can repel olfactory bulb axons in the embryo (Li et al., 1999; Nguyen Ba-Charvet et al., 1999) and can also repel precursors of interneurons in the postnatal olfactory bulb (Hu, 1999; Wu et al., 1999). In the visual system, Slit proteins are repellents for axons of the retinal ganglion cells (Erskine et al., 2000; Niclou et al., 2000; Ringstedt et al., 2000). In neocortical development, Slit plays an important role by repelling GABAergic neurons that migrate from the striatal primordium to the neocortex (Zhu et al., 1999).

A receptor for Slit is Roundabout (Robo), which was identified in Drosophila and Caenorhabditis elegans for its role in axon guidance (Kidd et al., 1998; Zallen et al., 1998). Further analysis in Drosophila indicates that robo interacts genetically with slit (Kidd et al., 1999). In vitro biochemical studies have shown that Slit and Robo proteins interact biochemically (Brose et al., 1999; Li et al., 1999; Yuan et al., 1999). Cell surface binding shows that the secreted Slit protein can bind to the surface of cells expressing Robo (Li et al., 1999; Chen et al., 2000).

Both Slit and Robo are large proteins. After the signal sequence at the $\mathrm{N}$ terminus of a typical Slit protein, there are four leucine-rich repeats, each surrounded by an N-terminal and a C-terminal flanking region (Rothberg et al., 1988, 1990). In Drosophila Slit, there are seven EGF repeats (Rothberg et al., 1988, 1990), whereas in all vertebrate Slits identified so far, there are nine EGF repeats (Holmes et al., 1998; Itoh et al., 1998; Nakayama et al., 1998; Brose et al., 1999; Li et al., 1999; Yuan et al., 1999). There is a laminin G domain (previously also known as the ALPS domain) followed by a cysteine-rich C-terminal region. Robo is a transmembrane (TM) protein with five Ig domains, three fibronectin type III repeats (FNIII), a TM domain, and four conserved cytoplasmic motifs in its intracellular domain (Kidd et al., 1998; Zallen et al., 1998). 
Biochemical purification shows that a fragment containing the N-terminal part, including the leucine-rich regions (LRRs) and four EGF repeats, was responsible for promoting axon branching (Wang et al., 1999). Because of the existence of multiple domains in each Slit protein, we were interested in determining the domains involved in axon guidance and neuronal migration. In particular, we wanted to address whether the same domain or domains are required for both axon guidance and neuronal migration. Therefore, we have undertaken biochemical studies to dissect the domains in Slit and Robo for protein-protein interactions and tested different fragments of Slit protein in functional assays of axon guidance and neuronal migration. Our results demonstrate directly that the LRRs in Slit are sufficient to act as a chemorepellent for projecting axons and migrating neurons and suggest indirectly that the EGF repeats are involved in controlling the diff usion of Slit. The finding that the same region in the Slit protein functions to guide axons and neurons supports the idea of a conserved mechanism between axon guidance and neuronal migration (Wu et al., 1999).

\section{MATERIALS AND METHODS}

Plasmids. A myc-tagged human Slit2 (hSlit) protein (hSlit2-myc) was expressed by a pCS2+-based construct with the full-length human Slit2 fused at the $\mathrm{C}$ terminus with six copies of the myc epitope. hSlit1076-myc and hSlit890-myc were made by fusing N-terminal 1076 amino acid (aa) residues and N-terminal 890 aa residues to the myc epitope. hSlitCR1myc was made to express the signal peptide followed by the $\mathrm{C}$-terminal region from aa 892 until the $\mathrm{C}$ terminus at aa 1529. Constructs have also been made to express Xenopus Slit fragments; these constructs include xSlit N882 and C1, which are similar to hSlit890 and C1, respectively.

To express Robo as an epitope-tagged protein, the rat Robo1 (rRobo1) coding region was obtained by PCR using rat spinal cord cDNA and was then inserted into a pCS2 + vector containing a hemagglutinin (HA) epitope. RoboIg construct was made to express the five Ig repeats followed by the transmembrane and intracellular domains. RoboFN construct was made to express the three FNIII repeats followed by the transmembrane and intracellular domains. In both cases, the HA epitope was also tagged at the $\mathrm{C}$ terminus.

To make constructs for stable transfection, cDNA fragments encoding hSlit2, hSlit1076, hSlit890, and hSlitCR1 were inserted into a modified pIRESneo (Clontech, Palo Alto, CA), which contains the neomycin gene after an internal ribosomal entry site.

The plasmids were verified by sequencing at junction and were tested for expression of the corresponding proteins by using coupled in vitro transcription-translation.

Cell culture, transfection, and selection of stable cell lines. Human embryonic kidney (HEK) 293 cells were maintained in 10\% fetal bovine serum in DMEM (Life Technologies, Grand Island, NY). Cells were grown to $70 \%$ confluence on $10 \mathrm{~cm}$ tissue culture plates and transfected with $\sim 25 \mu \mathrm{g}$ of plasmid DNA per plate using calcium phosphate for 16-24 hr. GFP-pGL, a plasmid expressing the green fluorescent protein, was used to monitor transfection efficiency.

For stable transfection, linearized plasmids and their corresponding vector controls were transfected into HEK cells. Antibiotics were added 36-48 hr after transfection; selection was performed for 3 weeks with the media changed every $3 \mathrm{~d}$. A total of $300 \mu \mathrm{g} / \mathrm{ml} \mathrm{G} 418$ (Life Technologies) was used to select for hSlit2-myc, hSlit1076-myc, hSlit890-myc, or hSlitCR1-myc stable lines. Stable cell lines expressing hSlit2-myc, hSlit1076-myc, hSlit890-myc, or hSlitCR1-myc were obtained after isolating individual colonies and testing for protein expression by Western blotting and immunocytochemical staining with the anti-myc antibody.

Immunoprecipitation. For immunoprecipitation, plasmids encoding hSlit2-myc, hSlit1076-myc, hSlit890-myc, hSlitCR1-myc, or Robo1-HA, RoboIg-HA, RoboFN-HA, or control vectors were transfected into HEK cells. Conditioned media from cells transfected with hSlit2-myc, hSlit1076-myc, hSlit890-myc, hSlitCR1-myc, or the control vector were collected 72 or $96 \mathrm{hr}$ after transfection and concentrated using a Biomax$100 \mathrm{~K}$ or Biomax-30K ultrafree filter (Millipore, Bedford, MA). Lysates from cells transfected with Robo1-HA, RoboIg-HA, RoboFN-HA, or control vector were prepared with lysis buffer $(0.5 \%$ NP-40, $50 \mathrm{~mm}$ Tris,
pH 7.5, $150 \mathrm{~mm} \mathrm{NaCl}, 1 \mathrm{~mm}$ EDTA, $50 \mathrm{~mm} \mathrm{NaF}, 1 \mathrm{~mm} \mathrm{Na}_{3} \mathrm{VO}_{4}, 1 \mathrm{~mm}$ DTT, $1 \mu \mathrm{mm}$ PMSF, $25 \mu \mathrm{g} / \mathrm{ml}$ leupeptin, $25 \mu \mathrm{g} / \mathrm{ml}$ aprotinin, and 150 $\mu \mathrm{g} / \mathrm{ml}$ benzamidine). Conditioned media containing hSlit2-myc, hSlit1076-myc, hSlit890-myc, hSlitCR1-myc, or control medium were mixed with lysates from Robo1-HA, RoboIg-HA, RoboFN-HA, or control cells, respectively. Immunoprecipitation was performed as described previously (Li et al., 1999).

Culture of olfactory bulb explants, anterior subventricular zone explants, and brain slices. Coculture of olfactory bulb explants with HEK cell aggregates was done according to a protocol described by Li et al. (1999). Quantification of axon projection from the olfactory bulb explants was performed according to the diagram shown in Figure $3 F$, which is modified from Keynes et al. (1997), their Figure $1 B$

Coculture of anterior subventricular zone (SVZa) explants with HEK cell aggregates was described by Wu et al. (1999). Semiquantification of cell migration was carried to the diagram shown in Figure $5 F(\mathrm{Wu}$ et al., 1999; Zhu et al., 1999).

Brain slice and whole-mount culture. Sagittal sections of $300 \mu \mathrm{m}$ were made by a vibratome; those containing the SVZa, the rostral migratory stream (RMS), and the olfactory bulb were used. A small piece of DiI (Molecular Probes, Eugene, OR) crystal was inserted into the SVZa. The slices were cultured in a manner similar to that described previously (Wu et al., 1999). HEK cells expressing hSlit2, hSlit1076, hSlit890, or hSlitCR1 or control HEK cells were labeled with 3,3' dioctadecyloxacarbocyanine (DiO) (Molecular Probes). A thin layer of cell aggregates was trimmed to $100-200 \mu \mathrm{m}$ in length and then overlaid on the top of the RMS. DiI and DiO signals were visualized with different filters under a microscope. Images were recorded with a Spot camera (Diagnostic Instruments, Sterling Heights, MI).

Whole-mount preparations of olfactory bulb-telencephalon coculture were performed with a protocol similar to that described by $\mathrm{Wu}$ et al. (1999).

\section{RESULTS}

\section{Domains in Slit and Robo involved in protein-protein interactions}

Three slit genes and three robo genes have been identified in mammals (Holmes et al., 1998; Itoh et al., 1998; Nakayama et al., 1998; Brose et al., 1999; Li et al., 1999; Yuan et al., 1999). Although their patterns of expression are different, they have not been found to differ biochemically or functionally. Proteins from one species also function in other species. Thus, in our previous studies, Xenopus Slit binds to mammalian Robo and is active on avian and mammalian tissues. To dissect the domains in Slit and Robo proteins for biochemical interactions, we have used hSlit2 and rRobo1.

A diagram of hSlit 2 is shown in Figure $1 A$. We made constructs expressing different portions of hSlit 2 as fusion proteins tagged at the $\mathrm{C}$ terminus with six myc epitopes (Fig. $1 A$ ). hSlit1076 contains the N-terminal 1076 aa residues including the four LRRs and four EGF repeats. HSlit1076 is slightly shorter than the N-terminal fragment identified by Wang et al. (1999), which would correspond to the N-terminal 1121 aa in the hSlit2 used in our experiments. hSlit890 contains only the four LRRs. Deletion of the four LRRs from the full-length hSlit2 results in hSlitCR1, which contains all nine EGF repeats, the laminin $\mathrm{G}$ domain, and the C-terminal cysteine-rich domain. After constructs encoding the full length hSlit2, hSlit1076, hSlit890, hSlitCR1, or the control vector were separately transfected into HEK cells, conditioned media were collected. Western blot analysis shows that proteins of the expected length were secreted extracellularly into the culture medium (Fig. 1C).

A diagram of the full-length rRobo1 is shown in Figure $1 B$. RoboIg contains five Ig domains, but not the three FN III repeats in the extracellular part. RoboFN contains the three FNIII repeats in the extracellular part. Both RoboIg and RoboFN have the transmembrane and the intracellular domains and are tagged 
A hSlit2

hSlit1076

hSlit890

hSlitCR1
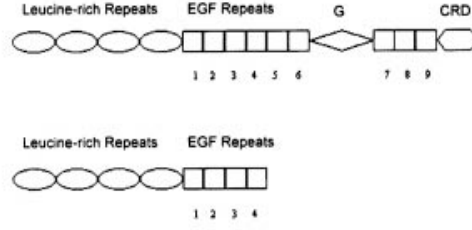

123456,10

3

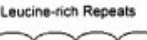

B

Robo

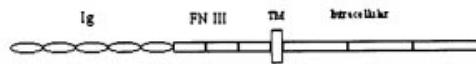

RoboIg

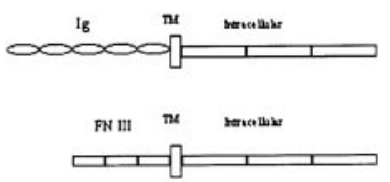

RoboFN

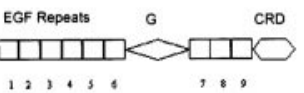

C

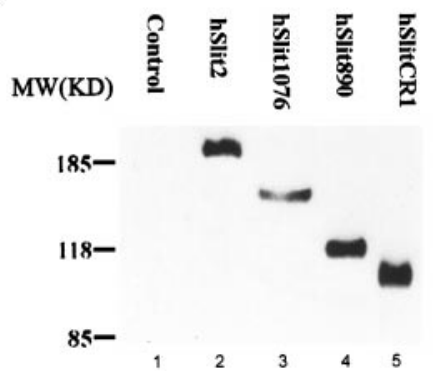

D

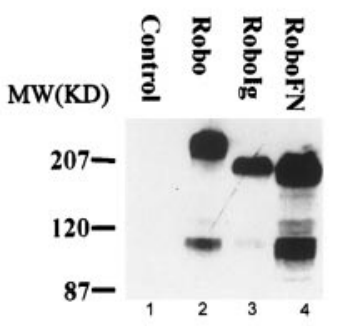

Figure 1. Expression of hSlit2 and aRobo1 proteins in HEK cells. A, A diagram of the full-length human Slit2 protein and its fragments. The signal sequence is included by the constructs expressing these proteins. Leucine-rich regions are represented by the ovals. EGF repeats are represented by squares. $G$, Laminin $\mathrm{G}$ domain (or the ALPS domain); $C R D$, cysteine-rich C-terminal domain. $B$, A diagram of the full-length rat Robo1 protein and its fragments. Ig, Ig domains. $C$, Expression of different Slit fragments by HEK cells. Proteins secreted into the extracellular medium were detected by an antibody recognizing the myc epitope at the $\mathrm{C}$ terminus of all fragments. $D$, Expression of different Robo1 proteins in HEK cells. Proteins in the lysates of HEK cells were detected by an anti-HA antibody recognizing the HA epitope of all fragments.

Figure 2. Biochemical interaction between Slit and Robo. A, Binding of the full-length Robo to different Slit proteins. Immunoprecipitation with the anti-HA antibody and Western blot analysis with the anti-myc antibody showed that Robo was able to bind to the full length and to the N-terminal 1076 and 890 aa residues but not to the C-terminal CR1 of Slit or to control HEK medium. Equal amounts of proteins were loaded for different fragments of Slit. $B$, Binding of RoboIg protein to different Slit proteins. Robolg could bind to hSlit2, hSlit1076, and hSlit890 but not to hSlitCR1 or to control HEK medium. The anti-HA antibody was used to precipitate the RoboIg protein, and the anti-myc antibody was used to detect the Slit proteins. $C$, Binding of RoboFN protein to different Slit proteins. RoboFN could not bind to hSlit2, hSlit1076, hSlit890, hSlitCR1, or control HEK medium. Anti-HA antibody was used to precipitate the RoboFN protein, and the anti-myc antibody was used to detect the Slit proteins.
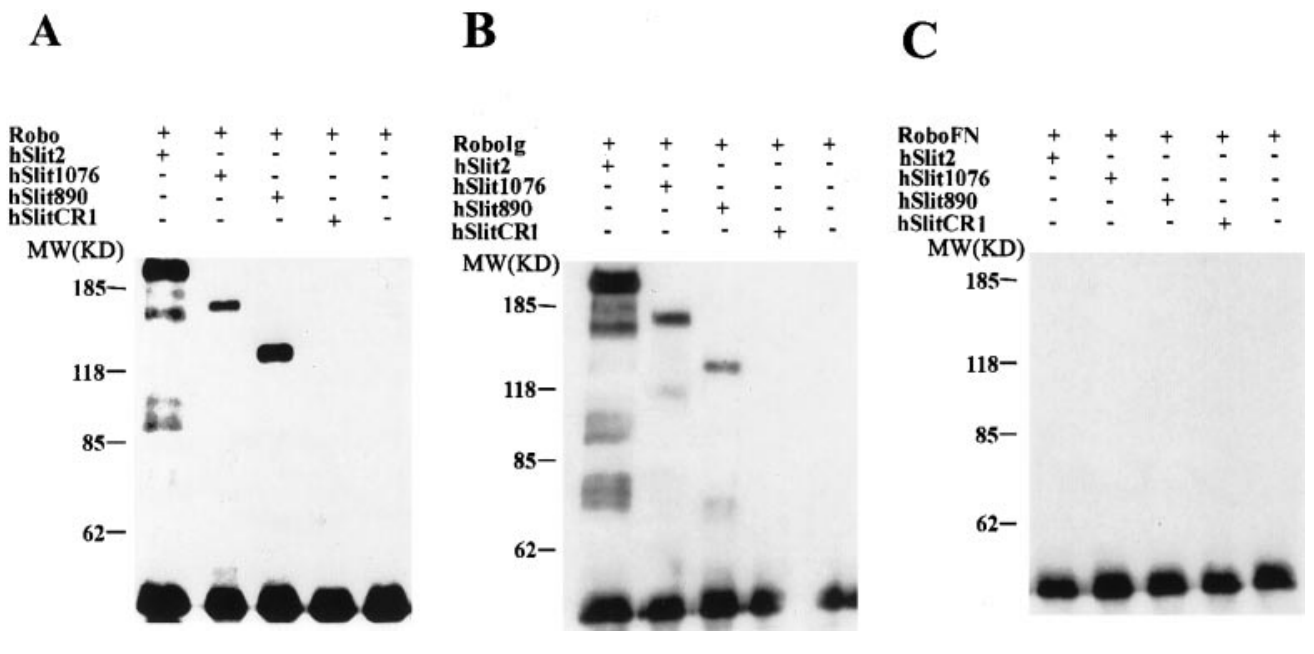

with an HA epitope at the $\mathrm{C}$ terminus. After constructs encoding the full-length rRobo1, RoboIg, RoboFN, or the control vector were separately transfected into HEK cells, lysates from the transfected HEK cells were prepared. Western blot analysis showed an upper band in the lysate of each transfection that corresponded to the expected protein (Fig. 1D). As observed previously ( $\mathrm{Li}$ et al., 1999), a lower band was present in cells transfected with rRobo1, confirming that Robo protein could be cleaved (Fig. 1D). The findings of the lower band in RoboIg and RoboFN indicate that the cleavage site is located in either the transmembrane domain or the intracellular domain because these two parts are present in all three Robo constructs 

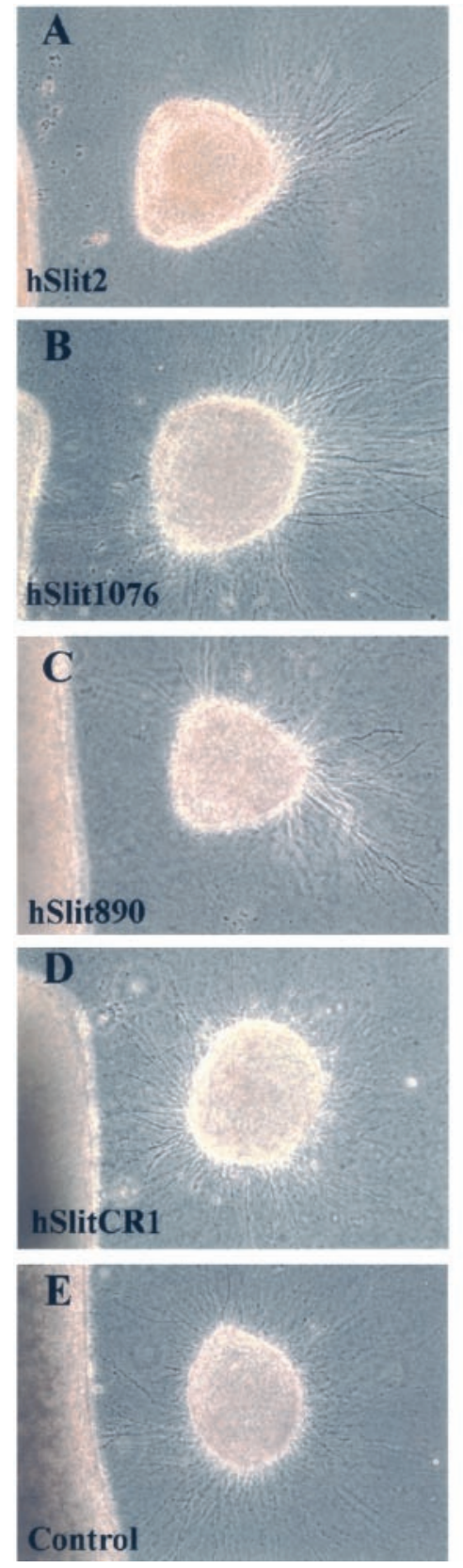

$\mathbf{F}$

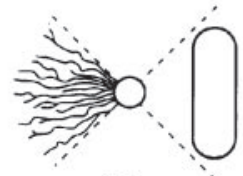

10

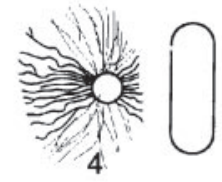

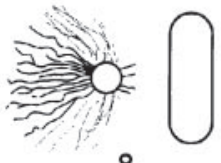

8
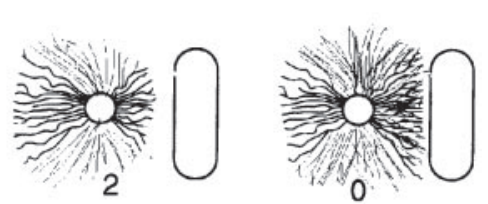

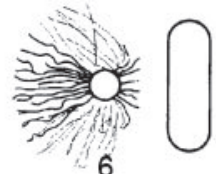

6
G

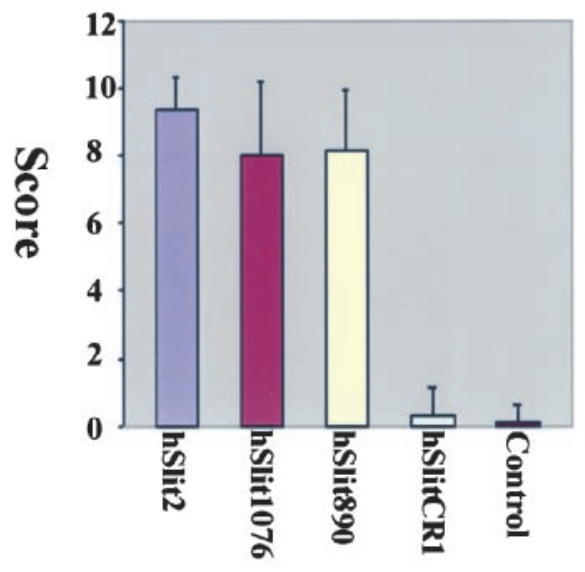

Figure 3. Chemorepulsive activities of Slit proteins on olfactory bulb axons growing in collagen gels. $A$, Distribution of axons growing from olfactory bulb explants after being cocultured with aggregates of HEK cells expressing hSlit2 in the collagen gel. $B$, Distribution of axons growing from olfactory bulb explants after being cocultured with aggregates of HEK cells expressing hSlit1076 in the collagen gel. $C$, Distribution of axons growing from olfactory bulb explants after being cocultured with aggregates of HEK cells expressing hSlit890 in the collagen gel. $D$, Distribution of axons growing from olfactory bulb explants after being cocultured with aggregates of HEK cells expressing hSlitCR1 in the collagen gel. E, Distribution of axons growing from olfactory bulb explants after being cocultured with aggregates of control HEK cells in the collagen gel. $F$, A diagram of the scheme used to semiquantify axon projections (modified from Keynes et al., 1997). $G$, Relative repulsive activities of Slit fragments. Explants were scored according to the scheme in $F$.
(Fig. 1D). The precise site of cleavage and its in vivo significance remains to be established.

To determine the domain of hSlit2 involved in binding to Robo1, the conditioned media from cells transfected with hSlit2, hSlit1076, hSlit890, hSlitCR1, or the control vector were separately incubated with lysates from cells transfected with the fulllength rRobo1 (Fig. 2A). Immunoprecipitation was performed as described previously (Li et al., 1999) using an anti-HA antibody. Precipitated proteins were detected by an anti-myc antibody. The full-length Robo1 protein could bind to the full-length hSlit2 (Fig. 2A, lane 1), hSlit1076 (Fig. 2A, lane 2), and hSlit890 (Fig. $2 A$, lane 3 ) protein. Robo1 did not bind to hSlitCR1 (Fig. $2 A$, lane 4 ). These results indicate that the four LRRs in Slit are sufficient for binding to the full-length Robo.

To determine the domains in rRobo1 involved in binding to hSlit2, the conditioned media from hSlit2, hSlit1076, hSlit890, hSlitCR1, or the control vector-transfected cells were separately incubated with lysates of cells transfected with RoboIg (Fig. 2B) or RoboFN (Fig. 2C). Immunoprecipitation was performed using the anti-HA antibody, and the precipitated proteins were detected by the anti-myc antibody. RoboIg could bind to the fulllength hSlit2 (Fig. 2B, lane 1), hSlit1076 (Fig. 2B, lane 2), and hSlit890 (Fig. 2B, lane 3). RoboIg protein did not bind to hSlitCR1 (Fig. 2B, lane 4). By contrast, RoboFN could not bind any forms of hSlit2 (Fig. $2 C$ ). These results indicate that the Ig domains of Robo protein are sufficient for binding to Slit.

Together, our results show that the LRRs in Slit and the Ig domains in Robo are necessary and sufficient for protein-protein interactions between Slit and Robo.

\section{The LRRs of Slit are sufficient for axon guidance both in vitro and in vivo}

We and others have shown previously that Slit is repulsive to axons projecting from the embryonic olfactory bulb ( $\mathrm{Li}$ et al., 

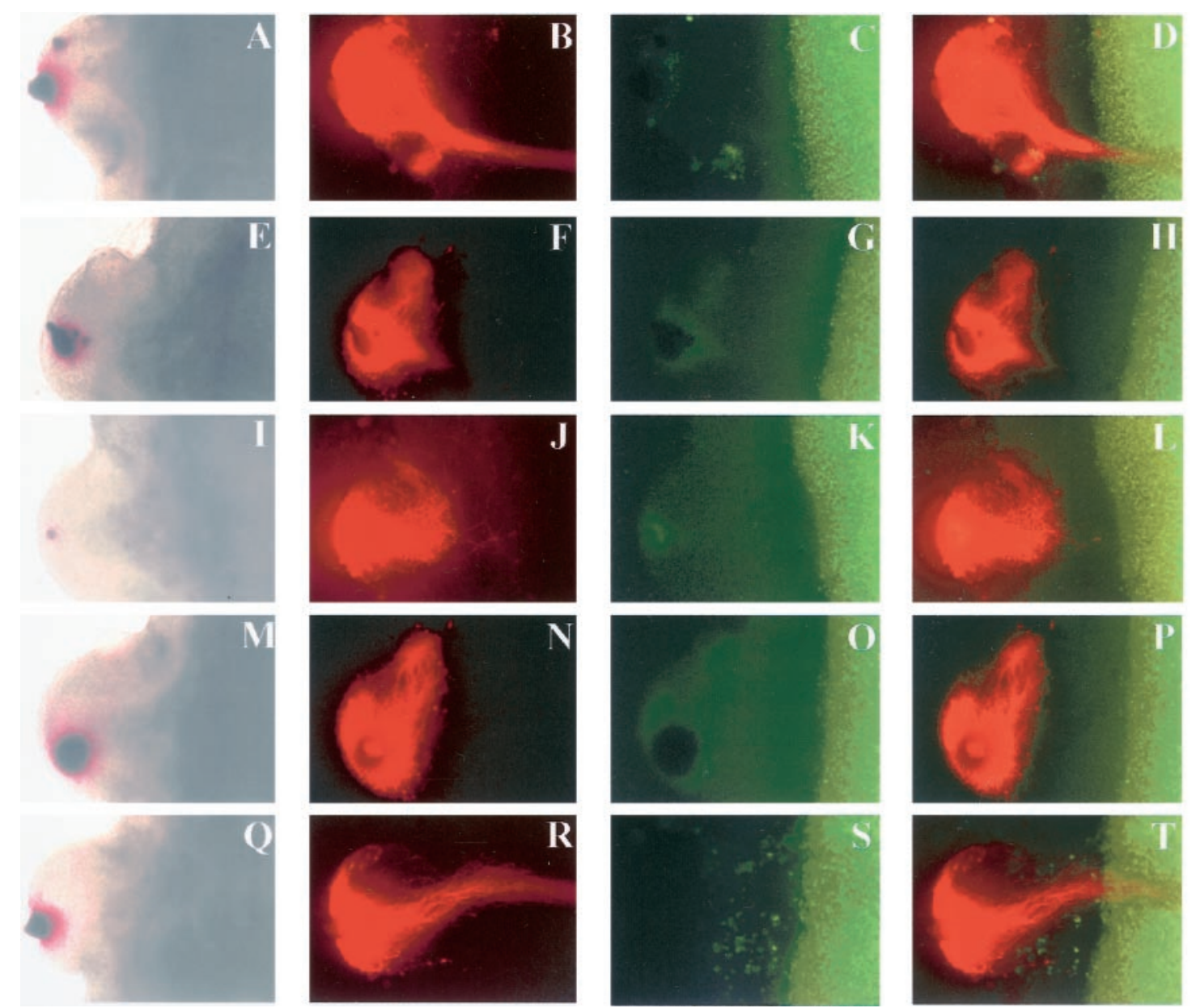

\section{Control}
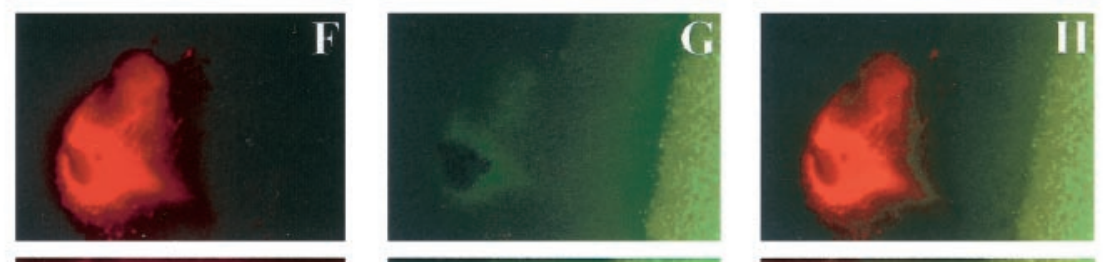

hSlit2
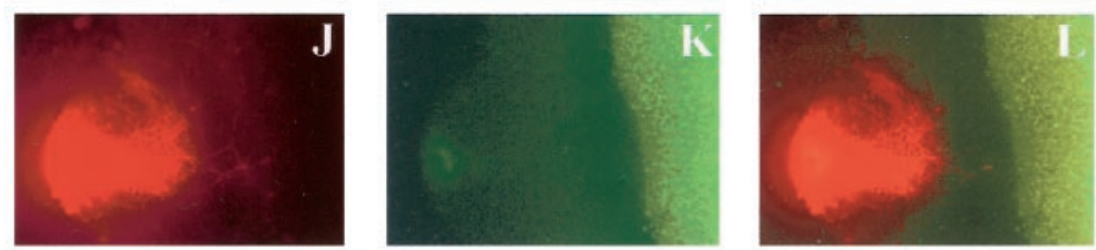

\section{hSlit1076}
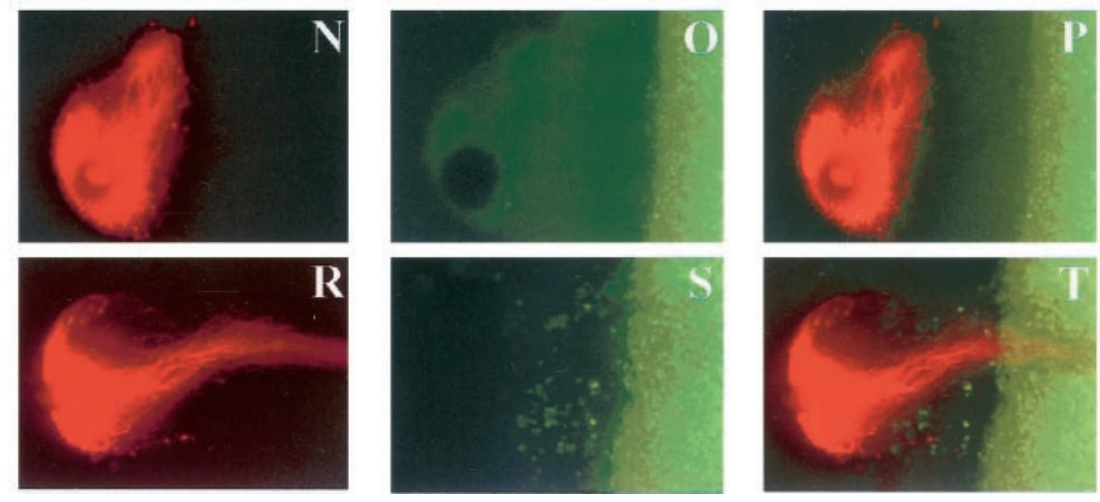

\section{hSlit890}

hSlitCR 1

Figure 4. Chemorepulsive activities of Slit proteins on olfactory bulb axons growing in the telencephalon. DiI crystals were placed into the olfactory bulb to label the axons (red in $B, F, J, N, R$ ) that project to the olfactory cortex. Aggregates of control HEK cells or HEK cells expressing different Slit proteins were labeled with $\mathrm{DiO}$ ( green in $C, G, K, O, S$ ) and laid on the top of the telencephalon. $D, H, L, P$, T, Composites of the red and green images. $A-D$, An aggregate of control HEK cells was laid on the top of the telencephalon, and the olfactory bulb axon was projected toward the olfactory cortex. $E-H$, The presence of an aggregate of HEK cells expressing hSlit2 prevented the projection of olfactory bulb axons. $I-L$, The presence of an aggregate of HEK cells expressing hSlit1076 prevented the projection of olfactory bulb axons. $M-P$, The presence of an aggregate of HEK cells expressing hSlit890 prevented the projection of olfactory bulb axons. $Q-T$, The presence of an aggregate of HEK cells expressing hSlitCR1 allowed the projection of olfactory bulb axons toward the olfactory bulb.

1999; Nguyen Ba-Charvet et al., 1999). To examine the domains of Slit that are functionally important for axon guidance, we first tested the activities of Slit fragments in collagen gels.

Olfactory bulb explants were isolated from embryonic day 13 mouse embryos and cocultured with either control HEK cells or HEK cells expressing the full-length form or the truncation mutant forms of Slit. When cocultured with control cells, axons grew symmetrically from the circumference of the olfactory bulb explants (with 4 of 54 explants showing slightly asymmetric axon outgrowth) (Fig. 3E). When cocultured with cells expressing hSlit2, olfactory bulb explants sent out axons asymmetrically, with more axons growing on the side distal to the Slit-expressing cells than on the side proximal to the Slit-expressing cells (Fig. $3 A$ ) (36 of 36 were asymmetric; $p<0.001$ when compared with the control; $\chi^{2}$ test). These results with hSlit 2 were similar to our previous results obtained with Xenopus Slit (Li et al., 1999). hSlit1076 was repulsive to the olfactory bulb axons (see Fig. $5 B$ ) ( 81 of 84 were asymmetric; $p<0.001$ ). hSlit890 was also repulsive to the olfactory bulb axons (see Fig. 5C) (101 of 103 were asymmetric; $p<0.001)$. In contrast, hSlitCR1 was not repulsive to the olfactory bulb axons (Fig. 3D) (6 of 54 were asymmetric; $p=$ $0.49)$. Quantitative analyses were performed with a scheme similar to that used in Keynes et al. (1997) (Fig. 3F); hSlit2, hSlit1076, and hSlit890 were clearly repulsive, whereas hSlitCR1 was not (Fig. $3 G$ ).

These results indicate that the LRRs present in hSlit890 were sufficient for guiding axons growing from olfactory bulb explants in collagen gels. To determine whether this conclusion is valid for olfactory bulb axons growing in their native environment in the telencephalon, we used the whole-mount assay, in which the olfactory bulb together with the telencephalon was isolated and cultured (Li et al., 1999).

The telencephalon region was covered with aggregates of control HEK cells or with HEK cells expressing hSlit2, hSlit1076, hSlit890, or hSlitCR1. All HEK cells were labeled with DiO before being placed on the telencephalon and could therefore be visualized under a fluorescence microscope (Fig. 4C, G, K, O,S). After $40 \mathrm{hr}$ in culture, a small piece of the lipophilic dye DiI was inserted into the olfactory bulb to reveal its projecting axons (Fig. $4 B, F, J, N, R)$. Axons could grow into the telencephalon covered 

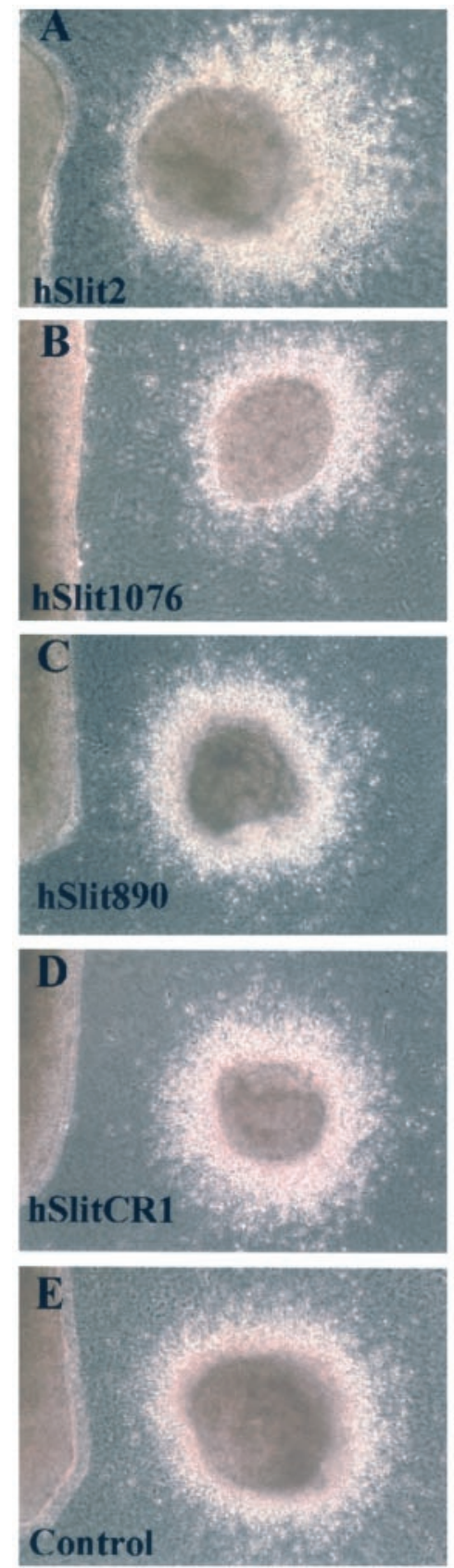

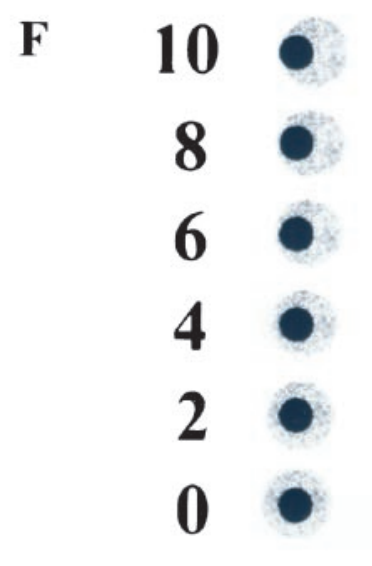

Scoring

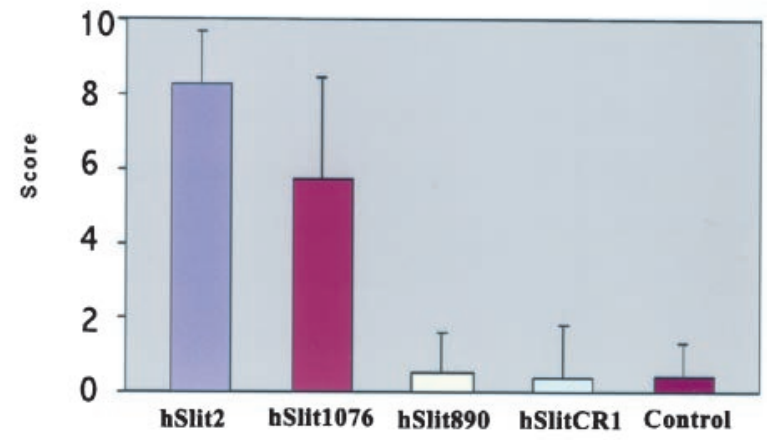

Figure 5. Chemorepulsive activities of Slit proteins on SVZa neurons migrating in collagen gels. $A$, Distribution of neurons migrating from an SVZa explant after being cocultured with an aggregate of HEK cells expressing hSlit 2 in the collagen gel. The presence of more SVZa neurons in the distal quadrant than in the proximal quadrant indicates a repulsive effect of hSlit2 on SVZa neurons. $B$, Distribution of neurons migrating from an SVZa explant after being cocultured with an aggregate of HEK cells expressing hSlit1076 in the collagen gel. hSlit1076 is also repulsive. $C$, Distribution of neurons migrating from an SVZa explant after being cocultured with an aggregate of HEK cells expressing hSlit890 in the collagen gel. No repulsive or attractive effect was found for hSlit890 in the collagen gel. $D$, Distribution of neurons migrating from an SVZa explant after being cocultured with an aggregate of HEK cells expressing hSlitCR1 in the collagen gel. No repulsive or attractive effect was found for hSlitCR1 in the collagen gel. E, Distribution of neurons migrating from an SVZa explant after being cocultured with an aggregate of control HEK cells in the collagen gel. $F$, A diagram of the scheme used to semiquantify the effects of different Slit proteins (modified from Zhu et al., 1999). $G$, Relative repulsive activities of different Slit proteins in collagen gels. The activities were scored according to the scheme in $F$.

with the control HEK cells (Fig. $4 A-D$ ) (axons projecting in all 40 preparations). As expected from our previous studies (Li et al., 1999), axons turned away from the part of the telencephalon covered with HEK cells expressing hSlit2 (Fig. 4E-H) (40 of 40 preparations had axons repelled; $p<0.001$ when compared with the control; $\chi^{2}$ test). hSlit1076 repelled olfactory bulb axons (Fig. $4 I-L$ ) (38 of 40 repelled; $p<0.001$ ). hSlit890 also repelled olfac- tory bulb axons (Fig. $4 M-P$ ) (37 of 40 repelled; $p<0.001$ ). In contrast, axons could grow into the telencephalon covered with HEK cells expressing hSlitCR1 (Fig. 4Q-T) (2 of 40 repelled; $p>$ $0.5)$.

Together, our results indicate that the four LRRs are sufficient to repel olfactory bulb axons growing both in vitro in collagen gels and in vivo in the telencephalon. 

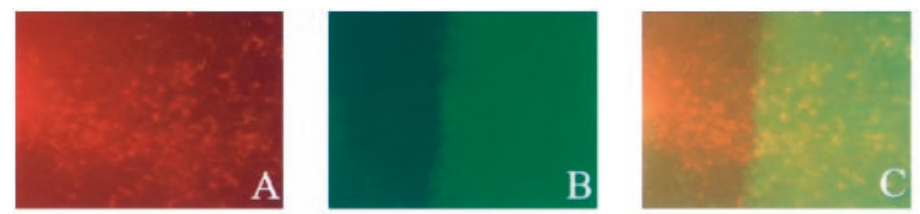

Control
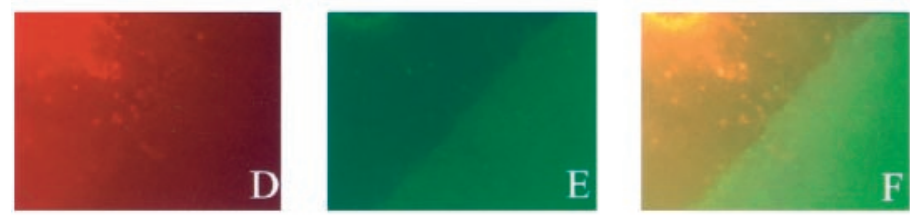
VZa neurons migrating in the RMS. DiI crystals were inserted into the SVZa in sagittal sections of postnatal migrating into the RMS. Aggregates of control HEK cells or HEK cells expressing different Slit proteins were labeled with $\mathrm{DiO}$ (green in $B, E, H, K, N$ ) and placed on the top of the RMS. $C, F, I, L, O$, Composites of the red and green images. Data in these panels could only be analyzed qualitatively (either with or without SVZa neurons in the RMS covered by the HEK aggregates); they could not be analyzed quantitatively because the numbers of SVZa neurons vary because of the precise size and insertion depth of DiI crystals. $A-C$, An aggregate of control HEK cells was placed on the top of the RMS and SVZa neurons migrated into the RMS. $D-F$, An aggregate of HEK cells expressing hSlit2 was placed on the top of the RMS and SVZa neurons failed to migrate into the RMS. $G-I$, An aggregate of HEK cells expressing hSlit1076 was placed on the top of the RMS and SVZa neurons failed to migrate into the RMS. $J-L$, An aggregate of HEK cells expressing hSlit890 was placed on the top of the RMS and SVZa neurons failed to migrate into the RMS. $M-O$, An aggregate of $\mathrm{HEK}$ cells expressing hSlitCR 1 was placed on the top of the RMS and SVZa neurons migrated into the RMS.
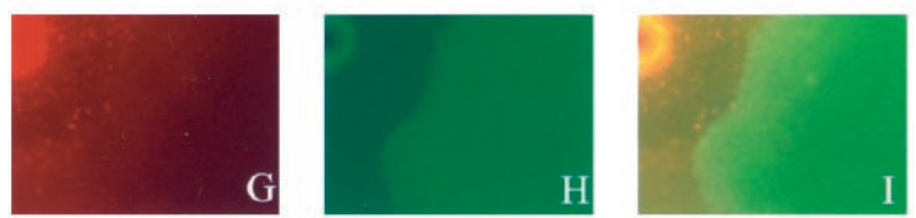

hSlit1076
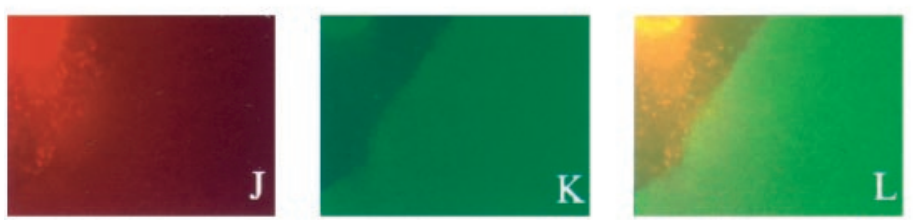

hSlit890
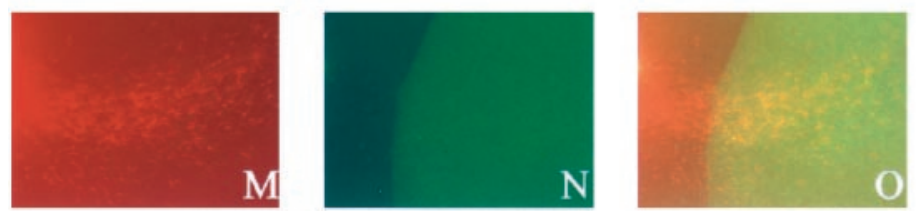

\section{The LRRs are sufficient to guide neuronal migration in the brain tissue but not in collagen gels}

Neuronal migration is an important process in neural development (Rakic, 1990; Hatten, 1999). Neurons migrating from the SVZa in the RMS to the olfactory bulb in neonatal rodents is an excellent model to study neuronal migration (Luskin, 1993; Lois et al., 1996; Goldman and Luskin, 1998). We and others have shown previously that $\mathrm{Slit} 2$ is repulsive to the $\mathrm{SVZ}$ a neurons $(\mathrm{Hu}$, 1999; Wu et al., 1999). Here we attempt to determine which domains in Slit are responsible for its guidance of neuronal migration.

We first tested the activities of hSlit2, hSlit1076, hSlit890, and hSlitCR1 in an in vitro collagen gel assay. In this assay, explants of SVZa were cocultured with aggregates of control HEK cells or HEK cells expressing different forms of hSlit2. As shown previously (Wu et al., 1999), cells migrated symmetrically out of SVZa explants when cocultured with control HEK cells (Fig. 5E) (9 of 48 explants had a slightly asymmetric distribution of migrating cells). When cocultured with hSlit2 aggregates, cells migrated asymmetrically from SVZa explants, with more cells in the distal quadrants than in the proximal quadrants (Fig. 5A) (118 of 118 explants with asymmetric migration; $p<0.001$ when compared with the control). HSlit1076 was also repulsive to the SVZa neurons (Fig. 5B) (69 of 88 with asymmetric migration; $p<$ 0.001). hCR1 was not repulsive to the SVZa neurons (Fig. $5 D)(8$ of 60 with weakly asymmetric migration; $p>0.5)$. The most surprising finding was that hSlit890 was not repulsive or attractive to SVZa neurons migrating in the collagen gel (Fig. 5C) (14 of 74 with weakly asymmetric migration; $p>0.5)$. To be more accu- rate, we used a semiquantitative scheme to estimate the activities of different forms of Slit (Fig. $5 F$ ). It was clear that hSlit2 and hSlit1076 were potent repellents for SVZa neurons, whereas hSlit890 and hSlitCR1 did not significantly affect SVZa migration in collagen gels (Fig. 5G).

These results indicate that the presence of four LRRs and four EGF repeats is sufficient for chemorepulsion of SVZa neurons. However, LRRs by themselves were not sufficient for repelling SVZa neurons in collagen gels. These findings could be explained by two possibilities: (1) there is a fundamental difference between the domains used in axon guidance and neuronal migration or (2) the requirement for additional EGF repeats in neuronal migration is simply because of diffusion in the collagen gel.

We then tested the activities of different forms of Slit on SVZa neurons migrating in their native pathway, the RMS. We had previously established a slice assay in which a sagittal section of rat embryonic brain containing the SVZa region, the RMS, and the olfactory bulb was preserved in culture (Wu et al., 1999). DiI crystals were inserted into the SVZa to trace the migration of neurons from the SVZa into the RMS (Wu et al., 1999). We placed HEK cells prelabeled with DiO on the RMS in the slices to examine their effects on cells migrating from the SVZa into the RMS (Fig. 6).

In slices cocultured with control HEK cells, $24 \mathrm{hr}$ after DiI labeling, migrating neurons were found in the RMS (Fig. 6A-C) ( 2 of 30 explants with no neurons in the RMS). When HEK cells expressing hSlit2 were placed on top of the slices, SVZa neurons failed to migrate into the RMS (Fig. $6 D-F$ ) (30 of 30 explants with no neurons in the RMS; $p<0.001$ when compared with the 
control; $\chi^{2}$ test). hSlit1076 was repulsive to SVZa neurons in the slices (Fig. 6G-I) (30 of 30 explants with no neurons in the RMS; $p<0.001)$. hSlitCR1 did not affect SVZa neuron migration into the RMS (Fig. 6M-O) (1 of 30 explants with no neurons in the $\mathrm{SVZa} ; p>0.5)$. However, hSlit890 was repulsive to SVZa neurons in the slices (Fig. $6 J-L$ ) (28 of 30 explants with no neurons in the RMS; $p<0.001)$. These results indicate that the four LRRs are sufficient for repelling SVZa neurons migrating in the RMS.

\section{DISCUSSION}

These studies have extended previous work on the role of Slit in axon guidance and neuronal migration. In addition to revealing the functional domains in Slit, the results presented here also support the idea that molecular mechanisms are conserved between axon guidance and neuronal migration (Wu et al., 1999). Using a construct that expresses the full-length Slit, we had suggested previously that Slit could function both in axon guidance and in neuronal migration (Wu et al., 1999). Because Slit was found to be proteolytically cleaved and only the N-terminal fragment was active in axon branching (Wang et al., 1999), it was possible that different fragments of Slit were used in axon guidance and neuronal migration. Results obtained here show that the N-terminal fragments with either 890 or 1076 aa are sufficient for both axon guidance and neuronal migration. Because no shorter fragments of Slit have been observed in vivo (Brose et al., 1999; Wang et al., 1999) or in vitro (Brose et al., 1999; Li et al., 1999; Chen et al., 2000), these results provide strong evidence that the same domains in Slit are responsible for both axon guidance and neuronal migration. Although previous genetic studies (Hedgecock et al., 1990) and functional studies with full-length proteins (Wu et al., 1999; Yee et al., 1999) have suggested conserved molecular mechanisms for axon guidance and neuronal migration, the present studies significantly strengthen that suggestion because these results rule out the possibility that different fragments of the same protein could be involved in axon guidance and neuronal migration.

We have found that the activities of hSlit1076 and hSlit890 were different when assayed in collagen gels for neuronal migration (Fig. 5). Because we have shown that a concentration gradient of the Slit protein is required for its guidance of neuronal migration (Wu et al., 1999), the simplest explanation for this difference is that the diffusion rates for these two forms are different. We suggest that the four EGF repeats present in hSlit1076 but absent in hSlit890 could affect protein diffusion. This could also explain why the behavior of hSlit890 in the collagen gel is different from its behavior in brain slices (Figs. 5, 6). Other explanations for the behavior of hSlit 890 such as efficiency of protein secretion in collage gels or the presence of synergistic activities in brain slices cannot be ruled out but are not as simple as the diffusion hypothesis to explain both the difference between axon guidance and neuronal migration in collagen gels and the difference in neuronal migration between the collagen gels and the brain slices.

Slit is a large protein with an apparent molecular weight of 200 kDa (Brose et al., 1999; Kidd et al., 1999; Li et al., 1999; Yuan et al., 1999; Chen et al., 2000). It was found that the full-length Slit protein is proteolytically cleaved into two fragments: a $140 \mathrm{kDa} N$-terminal fragment and a $55-60 \mathrm{kDa}$ C-terminal fragment (Brose et al., 1999; Wang et al., 1999). The cleaved fragment of Slit observed in vivo corresponds to the N-terminal 1121 aa in our hSlit2 sequence (Brose et al., 1999; Wang et al., 1999) and contains one EGF repeat more than the
hSlit1076 used in our studies. The fragment tested by Wang et al. (1999) was thus larger than the smallest fragment tested in our present studies, and the previous results were thus consistent with our suggestion that the N-terminal LRRs are sufficient for signaling. Because the branching activity is generally thought to be positive, whereas chemorepulsion is usually viewed as negative, it was not clear whether the same regions are used in these distinct activities. Our findings here that even shorter forms are functionally active in axon guidance and neuronal migration indicate that all functional activities reported so far are associated with the $\mathrm{N}$-terminal part.

We suggest that EGF repeats may be involved in controlling protein diffusion in vivo. Technically, our observation of different behaviors in collagen gels and brain slices reveals the limit of collagen gels and indicates that all activities of axon guidance and neuronal migration should be tested both in vitro and in vivo.

hSlitCR1 includes the C-terminal region, along with all the EGF repeats, the laminin $\mathrm{G}$ domain, and the $\mathrm{C}$-terminal cysteine rich region. We have found that hSlitCR1 does not bind to Robo and has no activities in vitro or in vivo on axon guidance or neuronal migration. It thus seems that the $\mathrm{C}$-terminal part is unlikely to play a sufficient role in signaling. Therefore, the function of all these domains is not known at present. The evolutionary conservation of these sequences suggests that they are also likely to play essential roles in vivo.

Although Slit has been shown to be able to guide axon projection and neuronal migration, we note that, so far, there is no evidence that endogenous Slit proteins are involved in axon projection and neuronal migration. Similarly, although the septum is repulsive to olfactory bulb axons (Pini, 1993), it has not been established that the septum plays a role in guiding the projection of the olfactory bulb in the embryo.

The intracellular domains of Robo have been studied recently for their important roles in binding to downstream signal transduction molecules (Bashaw and Goodman, 1999; Bashaw et al., 2000). Our studies of the domains in Robo indicate that only the Ig domains are required for its binding to Slit. Although the functional significance of the Ig and FNIII domains still needs to be investigated, these results provide a beginning to biochemically dissect the extracellular domains of Robo. Robo is cleaved in cultured HEK cells (Li et al., 1999; Chen et al., 2000). Results from the present series of experiments suggest that the cleavage site seems to be present in either the transmembrane or the intracellular domain. The physiological significance of this cleavage remains unknown.

There are other molecules involved in neuronal positioning. For example, the secreted protein Reelin is crucial for neuronal positioning and has been found to bind several transmembrane proteins (for review, see Rice and Curran, 1999). It will interesting to see what domains of Reelin are responsible for binding to these different transmembrane proteins and what domains are functionally crucial for neuronal positioning.

\section{REFERENCES}

Bashaw G, Goodman CS (1999) Chimeric axon guidance receptors: the cytoplasmic domains of Slit and Netrin receptors specify attraction versus repulsion. Cell 97:917-926.

Bashaw G, Kidd T, Murray D, Pawson T, Goodman CS (2000) Repulsive axon guidance: Abelson and Enabled play opposing roles downstream of the Roundabout receptor. Cell 101:703-715.

Battye R, Stevens A, Jacobs JR (1999) Axon repulsion from the midline of the Drosophila CNS requires slit function. Development 126:2475-2481.

Brose K, Bland KS, Wang KH, Arnott D, Henzel W, Goodman CS, Tessier-Lavigne M, Kidd T (1999) Slit proteins bind Robo receptors 
and have an evolutionarily conserved role in repulsive axon guidance. Cell 96:795-806.

Chen J, Wu W, Li HS, Fagaly T, Zhoul, Wu JY, Rao Y (2000) Embryonic expression and extracellular secretion of Xenopus Slit. Neuroscience 96:231-236.

Colamarino SA, Tessier-Lavigne M (1995) The role of the floor plate in axon guidance. Annu Rev Neurosci 18:497-529.

Culotti JG, Kolodkin AL (1996) Functions of netrins and semaphorins in axon guidance. Curr Opin Neurobiol 6:81-88.

Erskine L, Williams SE, Brose K, Kidd T, Rachel RA, Goodman CS, Tessier-Lavigne M, Mason CA (2000) Retinal ganglion cell axon guidance in the mouse optic chiasm: expression and function of Robos and Slits. J Neurosci 20:4975-4982.

Flanagan JG, Vanderhaeghen P (1998) The Ephrins and Eph receptors in neural development. Annu Rev Neurosci 21:309-345.

Frisen J, Holmberg J, Barbacid M (1999) Ephrins and their Eph receptors: multitalented directors of embryonic development. EMBO J 18:5159-5165.

Goldman SA, Luskin MB (1998) Strategies utilized by migrating neurons of the postnatal vertebrate forebrain. Trends Neurosci 21:107-114.

Hatten ME (1999) Central nervous system neuronal migration. Annu Rev Neurosci 22:511-539.

Hedgecock EM, Culotti JG, Hall DH (1990) The unc-5, unc-6, and unc-40 genes guide circumferential migrations of pioneer axons and mesodermal cells on the epidermis in C. elegans. Neuron 4:61-85.

Holder N, Klein R (1999) Eph receptors and ephrins: effectors of morphogenesis. Development 126:2033-2044.

Holmes GP, Negus K, Burridge L, Raman S, Algar E, Yamada T, Little MH (1998) Distinct but overlapping expression patterns of two vertebrate slit homologs implies functional roles in CNS development and organogenesis. Mech Dev 79:57-72.

$\mathrm{Hu} \mathrm{H}$ (1999) Chemorepulsion of neuronal migration by Slit2 in the developing mammalian forebrain. Neuron 23:703-711.

Itoh A, Miyabayashi T, Ohno M, Sakano S (1998) Cloning and expressions of three mammalian homologues of Drosophila slit suggest possible roles for Slit in the formation and maintenance of the nervous system. Mol Brain Res 62:175-186.

Kennedy TE, Serafini T, De la Torre JR, Tessier-Lavigne M (1994) Netrins are diff usible chemotropic factors for commissural axons in the embryonic spinal cord. Cell 78:425-435.

Keynes R, Tannahill D, Morgenstern DA, Johnson AR, Cook GM, Pini A (1997) Surround repulsion of spinal sensory axons in higher vertebrate embryos. Neuron 18:889-897.

Kidd T, Brose K, Mitchell KJ, Fetter RD, Tessier-Lavigne M, Goodman CS, Tear G (1998) Roundabout controls axon crossing of the CNS midline and defines a novel subfamily of evolutionarily conserved guidance receptors. Cell 92:205-215.

Kidd T, Bland KS, Goodman CS (1999) Slit is the midline repellent for the Robo receptor in Drosophila. Cell 96:785-794.

Li HS, Chen JH, Wu W, Fagaly T, Zhou L, Yuan W, Dupuis S, Jiang ZH, Nash W, Gick C, Ornitz DM, Wu JY, Rao Y (1999) Vertebrate slit, a secreted ligand for the transmembrane protein roundabout, is a repellent for olfactory bulb axons. Cell 96:807-818.

Lois C, Garcia-Verdugo J-M, Alvarez-Buylla A (1996) Chain migration of neuronal precursors. Science 271:978-981.

Luskin MB (1993) Restricted proliferation and migration of postnatally generated neurons derived from the forebrain subventricular zone. Neuron 11:173-189.

Mellitzer G, Xu Q, Wilkonson DG (2000) Control of cell behavior by signaling through Eph receptors and ephrins. Curr Opin Neurobiol 10:400-408.

Nakayama M, Nakajima D, Nagase T, Nomura N, Seki N, Ohara O
(1998) Identification of high-molecular-weight proteins with multiple EGF-like motifs by motif-trap screening. Genomics 51:27-34.

Nguyen Ba-Charvet KT, Brose K, Marillat V, Kidd T, Goodman CS, Tessier-Lavigne M, Sotelo C, Chedotal A (1999) Slit2-mediated chemorepulsion and collapse of developing forebrain axons. Neuron 22:463-473.

Niclou SP, Jia L, Raper JA (2000) Slit2 is a repellent for retinal ganglion cell axons. J Neurosci 20:4962-4974.

Nusslein-Volhard C, Wieschaus E, Kluding H (1984) Mutations affecting the pattern of the larval cuticle in Drosophila melanogaster. I. Zygotic loci on the second chromosome. Roux's Arch Dev Biol 193:267-282.

Pini A (1993) Chemorepulsion of axons in the developing mammalian central nervous system. Science 261:95-98.

Rakic P (1990) Principles of neural cell migration. Experientia 46:882-891.

Raper JA (2000) Semaphorins and their receptors in vertebrates and invertebrates. Curr Opin Neurobiol 10:88-94.

Raper JA, Tessier-Lavigne M (1999) Growth cones and axon pathfinding. In: Fundamental neuroscience (Zigmond M, Bloom FE, Landis SC, Roberts JL, Squire LE, eds), pp 519-546. New York: Academic.

Rice DS, Curran T (1999) Mutant mice with scrambled brains: understanding the signaling pathways that control cell positioning in the CNS. Genes Dev 13:2758-2773.

Ringstedt T, Braisted JE, Brose K, Kidd T, Goodman C, Tessier-Lavigne M, O'Leary DDM (2000) Slit inhibition of retinal axon growth and its role in retinal axon pathfinding and innervation patterns in the diencephalon. J Neurosci 20:4983-4991.

Rothberg JM, Hartley DA, Walther Z, Artavanis-Tsakonas S (1988) Slit: an EGF-homologous locus of D. melanogaster involved in the development of the embryonic central nervous system. Cell 55:1047-1059.

Rothberg JM, Jacob JR, Goodman CS, Artavanis-Tsakonas S (1990) Slit: an extracellular protein necessary for the development of midline glia and commissural axon pathways contains both EGF and LRR domains. Genes Dev 4:2169-2187.

Serafini T, Kennedy TE, Galko MJ, Mirzayan C, Jessell TM, TessierLavigne M (1994) The netrins define a family of axon outgrowthpromoting proteins homologous to C. elegans UNC-6. Cell 78:409-424.

Tessier-Lavigne M, Goodman CS (1996) The molecular biology of axon guidance. Science 274:1123-1133.

Van Vactor D, Lorenz LJ (1999) Neural development: the semantics of axon guidance. Curr Biol 9:R201-R204.

Wang KH, Brose K, Arnott D, Kidd T, Goodman CS, Henzel W, Tessier-Lavigne M (1999) Biochemical purification of a mammalian slit protein as a positive regulator of sensory axon elongation and branching. Cell 96:771-784.

Wu W, Wong K, Chen J, Jiang Z, Dupuis S, Wu JY, Rao Y (1999) Directional guidance of neuronal migration in the olfactory system by the protein Slit. Nature 400:331-336.

Yee KT, Simon HH, Tessier-Lavigne M, O'Leary DDM (1999) Extension of long leading processes and neuronal migration in the mammalian brain directed by the chemoattractant Netrin-1. Neuron 24:607-622.

Yuan W, Zhou L, Chen JH, Wu JY, Rao Y, Ornitz DM (1999) The mouse SLIT family: secreted ligands for ROBO expressed in patterns that suggest a role in morphogenesis and axon guidance. Dev Biol 212:290-306.

Zallen JA, Yi BA, Bargmann CI (1998) The conserved immunoglobulin superfamily member SAX3/Robo directs multiple aspects of axon guidance in C. elegans. Cell 92:217-227.

Zhu Y, Li HS, Zhou L, Wu JY, Rao Y (1999) Cellular and molecular guidance of GABAergic neuronal migration from the striatum to the neocortex. Neuron 23:473-485. 